\title{
Parasitic endometrioid adenomyoma on the bladder
}

\author{
Jiangtao Lyu' ${ }^{1} \cdot$ Chengzhi Zhao ${ }^{2}$
}

Received: 20 May 2021 / Accepted: 12 July 2021 / Published online: 9 August 2021

(c) The Author(s), under exclusive licence to Springer-Verlag GmbH Germany, part of Springer Nature 2021

A 50-year-old women (gravida 2, para 1, abortion 1) was admitted in hospital for adnexal mass found by transvaginal ultrasound for 2 years. She had no history of pelvic surgery. Because ultrasound failed to show the source of the mass, a MRI examination was taken. MRI revealed a mass measuring about $5.5 \times 4.1 \times 3.3 \mathrm{~cm}$, of low signal intensity in the T2-weighted images, suggesting with a broad ligament myoma or subserous myoma. On physical examination, a moving palpable mass was found in front of the uterus. At laparoscopy, the mass was found pedunculated to the bladder. There was no connection between it and the uterus. It had a well-circumscribed, subserous cystic mass with a thin wall containing old blood. It was resected and grinded up with a power morcellator in a bag. Eventually, the pathology result was uterine adenomyosis (Fig. 1).
Fig. 1 MRI revealed a mass between the bladder and uterus (A). The mass was found pedunculated to the bladder (B). The pathology result was uterine adenomyosis $(\mathbf{C})$
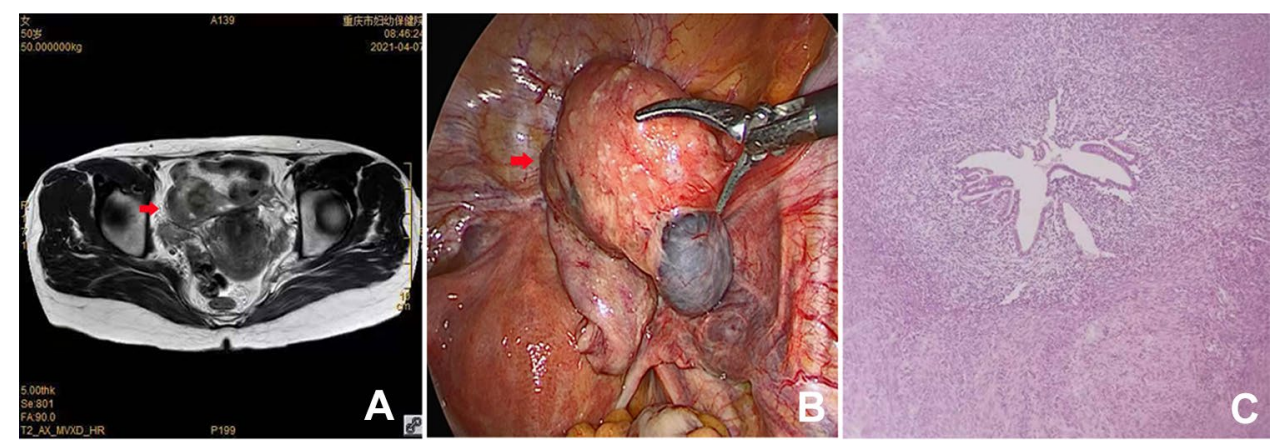

Chengzhi Zhao

330808042@qq.com

1 Department of Gynecologic Endocrinology, Chongqing Health Center for Women and Children, Chongqing, China

2 Department of Gynecologic Oncology, Chongqing Health Center for Women and Children, Chongqing, China
Author contributions JL contributed to writing. CZ contributed to case selection, data assessment and revising.

\section{Declarations}

Conflict of interest The author Jiangtao Lyu declares that he has no conflict of interest. The author Chengzhi Zhao declares that he has no conflict of interest.

Publisher's Note Springer Nature remains neutral with regard to jurisdictional claims in published maps and institutional affiliations. 